\title{
Local crystalline order in a 2D colloidal glass former
}

\author{
F. Ebert ${ }^{\mathrm{a}}$, P. Keim, and G. Maret \\ Fachbereich für Physik, Universität Konstanz, D-78457 Konstanz, Germany
}

\begin{abstract}
A mixture of two types of super-paramagnetic colloidal particles with long-range dipolar interaction is confined by gravity to a flat interface of a hanging water droplet. The particles are observed by video microscopy and the dipolar interaction strength is controlled by an external magnetic field. The local structure as obtained by pair correlation functions and bond order statistics is investigated as a function of system temperature and relative concentration. Although the system has no long-range order and exhibits glassy dynamics, different types of stable crystallites coexist. The local order of the globally disordered structure is explained by a small set of specific crystal structures. The statistics of crystal unit cells show a continuous increase of local order with decreasing system temperature as well as a dependence on sample history and local composition.
\end{abstract}

\section{Introduction}

Many systems show a dynamic arrest as revealed by a drastic increase of viscosity. This phenomenon of kinetic vitrification may take place even when a phase transition into a long-range ordered state is possible and eventually may occur under appropriate conditions; examples are monodisperse hard spheres [1], semicrystalline stacks of lamellar crystals in polymers [2] or binary mixtures in metal alloys [3].

It appears obvious that disordered structure and dynamics are related. One example for a close formal connection between structure and dynamics of a glass-forming system is provided by Mode Coupling Theory (MCT), since the only input into the MCT equations is the static structure factor $[4,5]$. Nevertheless, the microscopic connection between structure and dynamics is still under strong debate [6-8], especially the question how crystallization is connected to vitrification and dynamical heterogeneity $[9,10]$. Simulations $[10]$ suggest that crystallization plays a key role for the glass transition. The authors propose that "liquids tend to order into the equilibrium crystal, but frustration effects of locally lavored short-range ordering on long-range crystalline ordering prevent crystallization and help vitrification".

It was found in experiments [11], simulations [12], and theory [13] that glassy systems in 2D exhibit the full range of glass phenomenology known in three-dimensional glass formers, both in dynamics and structure. However, in experiments, glassy behavior in two dimensions is only pos-

\footnotetext{
a e-mail: florian.ebert@uni-konstanz.de
}

sible by introducing polydispersity [13]. The simplest form of polydispersity is that of a binary system, which may of course also turn into binary crystal structures.

In experiments, colloidal glasses have the advantage over atomic systems that, besides statistical averaged information about the local structure, "quasi-atomic" resolution is provided by microscopy $[6,11]$.

The binary colloidal 2D system investigated here consists of a mixture of particles with two different magnetic moments. It exhibits all typical phenomenological features of a glass former, e.g. drastic increase of relaxation times for increasing interaction strength, no long-range order, and dynamic heterogeneities [11]. The dynamics of the system was compared with MCT and good agreement was found [13]. Partial clustering of small particles was observed $[14,15]$ due to the nonadditivity of the dipolar binary potential. This leads to a heterogeneous distribution of particle composition, which results locally in a coexisting variety of small areas with different underlying crystal structures. Thus, heterogenous distribution of small particles suppresses long-range order. Extended stable crystal structures for $2 \mathrm{D}$ binary dipoles were found in $T=0$ lattice sum calculations $[16,17]$. Especially all locally ordered structures discussed in this paper were predicted to be stable.

The idea that the disordered structure of this system is made up from particular substructures (triangular structures) was originally discussed in [18]. However, in this paper we suggest that the local ordered structure originates from the tendency of the binary mixture towards crystallization. 


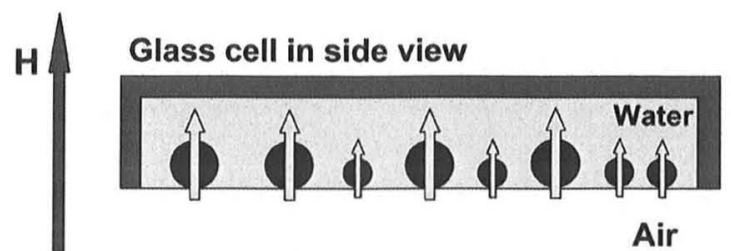

Fig. 1. Super-paramagnetic colloidal particles confined at a water/air interface due to gravity. The curvature of the interface is actively controlled to be completely flat; therefore the system is considered to be ideally two-dimensional. A magnetic field $\mathbf{H}$ perpendicular to the interface induces a magnetic moment $\mathbf{m}$ in each bead leading to a repulsive dipolar interaction.

Crystallization may be geometrically possible, provided the relative concentration matches a certain crystal structure. However, crystallization is not at odds with a glass transition in a binary system. A decrease in temperature can force a system into a dynamically arrested state due to strong increase of viscosity before crystallization can establish long-range order. Since the small particles cannot reorganize fast enough, much disorder is "frozen" in. As a consequence different competing crystalline structures appear, while the global structure remains amorphous.

\section{Experimental setup}

Our experimental setup consists of a mixture of two different kinds of spherical and super-paramagnetic colloidal particles (species A: diameter $d_{A}=4.5 \mu \mathrm{m}$, susceptibility $\chi_{A}=6.22 \cdot 10^{-11} \mathrm{Am}^{2} / \mathrm{T}$, density $\rho_{A}=1.5 \mathrm{~g} / \mathrm{cm}^{3}$ and species $\mathrm{B}$ : $d_{B}=2.8 \mu \mathrm{m}, \chi_{B}=6.6 \cdot 10^{-12} \mathrm{Am}^{2} / \mathrm{T}$, $\rho_{B}=1.3 \mathrm{~g} / \mathrm{cm}^{3}$ ) which are confined by gravity to a water/air interface. This interface is formed by a water drop suspended by surface tension in a top sealed cylindrical hole (6 mm diameter, $1 \mathrm{~mm}$ depth) of a glass plate as sketched in Figure 1. A magnetic field $\mathbf{H}$ is applied perpendicular to the water/air interface inducing a magnetic moment $\mathbf{M}=\lambda \mathbf{H}$ in each particle leading to a repulsive dipole-dipole pair interaction. Counterpart of the potential energy is thermal energy which generates Brownian motion. Thus the dimensionless interaction strength $\Gamma$ is defined by the ratio of the potential versus thermal energy:

$$
\begin{aligned}
\Gamma & =\frac{E_{\text {magn }}}{k_{B} T} \propto \frac{1}{T_{s y s}} \\
& =\frac{\mu_{0}}{4 \pi} \cdot \frac{\mathbf{H}^{2} \cdot(\pi \rho)^{3 / 2}}{k_{B} T}\left(\xi \cdot \lambda_{B}+(1-\xi) \lambda_{A}\right)^{2} .
\end{aligned}
$$

Here, $\xi=N_{B} /\left(N_{A}+N_{B}\right)$ is the relative concentration of small species with $N_{A}$ big and $N_{B}$ small particles and $\rho$ is the area density of all particles. The average distance of neighboring big particles is given by $l=1 / \sqrt{\rho}_{\text {big }}$. The interaction strength can be externally controlled by means of the magnetic field $H . \Gamma$ can be interpreted as an inverse temperature and controls the behavior of the system.
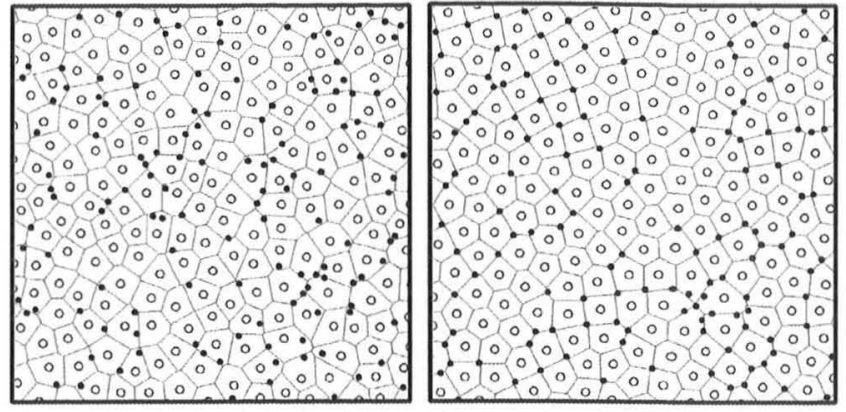

Fig. 2. Coordinates of big (open circles) and small particles (black discs) are shown for two values of $\Gamma$ (left: 5, right: 674). The Voronoi cells of only the big particles are sketched.

The ensemble of particles is visualized with video microscopy from below and the signal of a CCD 8-Bit grayscale camera is analyzed on a computer. The field of view has a size of $1158 \times 865 \mu \mathrm{m}^{2}$ containing typically $3 \cdot 10^{3}$ particles, whereas the whole sample contains about up to $10^{5}$ particles. Standard image processing is performed to get size, number and positions of the colloids. A computer controlled syringe driven by a micro-stage controls the volume of the droplet to get a completely flat surface. In this way fluctuations around the set-point of particle density are suppressed below $0.1 \%$ and the biggest observed particle-density gradient in the horizontal plane is less than $1 \%$. The latter is achieved by a variation of the inclination of the whole experimental setup. This inclination is also controlled actively by micro-stages with a resolution of $\alpha \approx 1 \mu \mathrm{rad}$. After several weeks of adjusting and equilibration this provides best equilibrium conditions for long-time stability. During data acquisition the images are analyzed with a frame rate down to $250 \mathrm{~ms}$. Trajectories for all particles in the field of view can be recorded over several days providing the whole phase space information. The thermal activated out-of-plane motion of the colloids is in the range of a few tenth of $\mathrm{nm}$ so the ensemble can be considered as ideally two-dimensional.

\section{Local order}

Adding small particles prevents global crystallization of the system. As the susceptibility of the small particles is roughly ten times smaller compared to that of the big ones the local structure is dominated by the latter. This is demonstrated in Figure 2 where the Voronoi cells of the big particles (small particles are ignored) are shown for two different interaction strengths $I$. Increasing $I$, the small particles are forced into the potential dips formed by the big particles. The Voronoi vertices of the big and the positions of the small particles are highly correlated. In Figure 3 the probability distribution of distances $d$ of the small particle to its next Voronoi vertex are shown for various temperatures. One sees that for increasing interaction strength the small particles are progressively pinned to the potential minima between the big ones. The deviation from a Gaussian distribution at higher interaction 


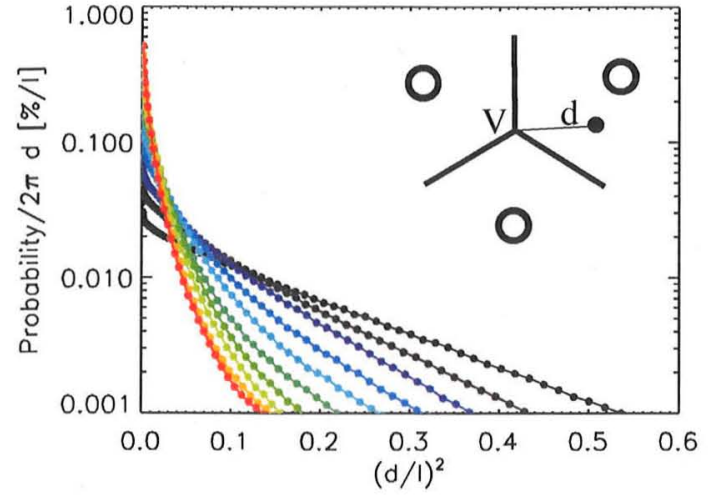

Fig. 3. The distribution of squared distances $d^{2}$ of small particles from their nearest Voronoi vertex $\mathrm{V}$ is plotted (see inset) for different values of $\Gamma$ (Color codes: dark to bright: 5, 16, $27,52,71,136,159,209,458,674)$. It is normalized with the circumference $2 \pi d$ and plotted on a logarithmic scale. A linear decay corresponds to a Gaussian distribution of the small particles around the vertices. With decreasing temperature the small particles are forced to the vertices.

strength originates from the fact that sometimes more than one particle is pinned near this minimum.

This behavior is analogous to that of a binary crystalline structure of purely repulsive particles, where the positions of particles are highly correlated such that the weaker interacting species are fixed to the potential minima of the big species. This tendency is at least found in all calculated stable $T=0$ crystal structures [16] for $\xi<0.5$. (For $\xi>0.5$ this is not true in general.)

\subsection{Formation of crystallites}

A direct motivation for the tendency towards crystallization is given by the observation of crystal grains such as those shown in Figure 4. Regions consisting only of big particles form hexagonal grains (Fig. 4A) as expected from monodisperse samples (melting temperature $\Gamma_{m} \approx$ $60[19,20])$. Here, interaction strengths are much higher $(\Gamma>220)$. Ensembles of many small particles do not exhibit crystalline order and form linear or branched chains (Fig. 4B). Again, this is due to their low susceptibility. To reach crystallization for the small particles alone, the magnetic field has to be stronger by a factor of 3.5 compared to the big particles [21]. Thus, monodisperse regions of small particles are expected to be fluid at the used magnetic fields and they are easily squeezed into chainlike structures by the surrounding big particles.

The highlighted particles in Figure $4 \mathrm{C}$ form a cluster of simple quadratic (SQ) unit cells made up of big particles with a twofold basis that contains one small particle in the center. Another example for this structure is given in Figure 4D where three touching crystal grains are shown. These crystallites are quite stable (over many days no melting has been observed, suggesting that an arrested ordering transition may have occurred). The pictures are
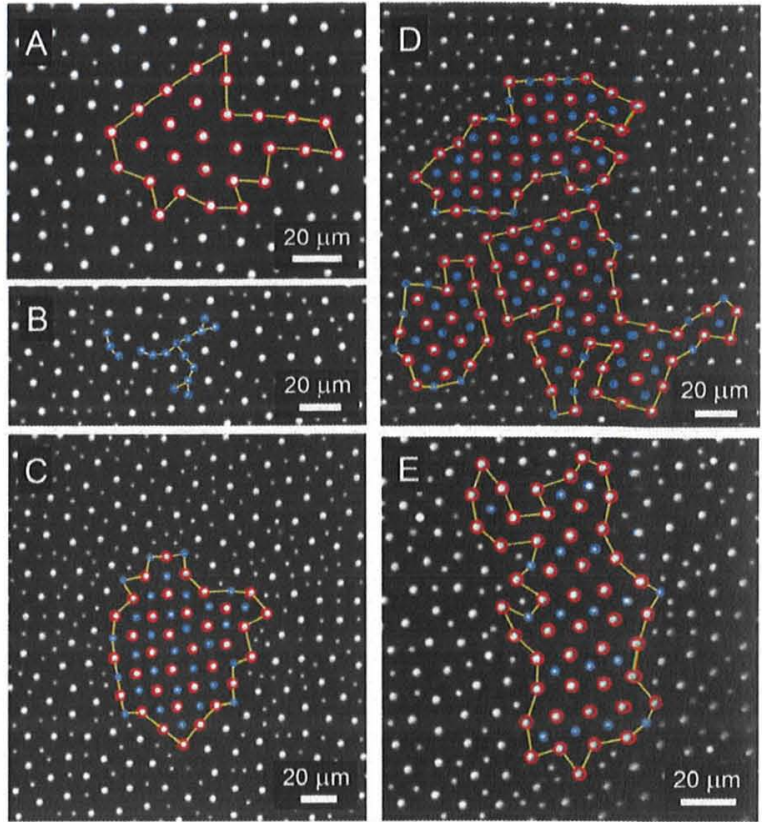

Fig. 4. Pictures A and B: Monodisperse structures of big and small particles at $I \approx 540$. Big particles form hexagonal crystal grains, while small particles only form chains. Picture C: SQ crystal grain consisting of 64 Particles at $\Gamma \approx 425$ with relative concentration $\xi=1 / 2$. Picture D: Three touching SQ crystal grains at $\Gamma \approx 540$. Picture E: A crystal grain at $\Gamma \approx 220$ consisting of SQ columns alternating with hexagonal rhombs. The relative concentration of this crystal structure is $\xi=1 / 3$. The average relative concentration of the samples in all shown snapshots was $\xi \approx 45 \%$.

representative examples for a very frequent appearance especially in a sample mixture close to $\xi=0.5$.

The example in Figure $4 \mathrm{E}$ is an alternating composition of SQ and hexagonal unit cells. The shown crystal structure is representative and was found several times but less often than pure SQ grains. The crystal structure can be described as rhombic [16] with a threefold basis of two big and one small particle. In this way the system can organize in a periodic structure with a relative concentration of $\xi=1 / 3$. However, the averaged relative concentration of the sample is $45 \%$.

The observed crystallites (up to 64 particles) cannot be formed by spontaneous fluctuations. An estimation of the probability for a spontaneous formation can be obtained by looking at classical nucleation theory [22]. The probabilities for such spontaneous formations are found to be diminutive [23]. This suggests that the frequent occurrence of crystallites is caused by the tendency towards crystallization which favors growth of equilibrium periodic structures.

Although the system shows glassy dynamics and is globally disordered [11], the local order reflects the underlying crystalline structure. In Figure 5 (right) the pair correlation functions for big and small particles are plotted in units of the average distance of big particles for a strongly 
Hexagonal

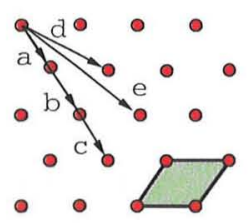

Simple Quadratic

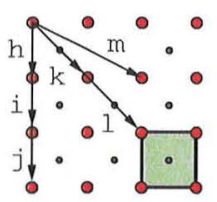

Chains

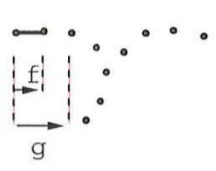

Rhombic

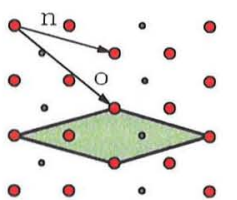

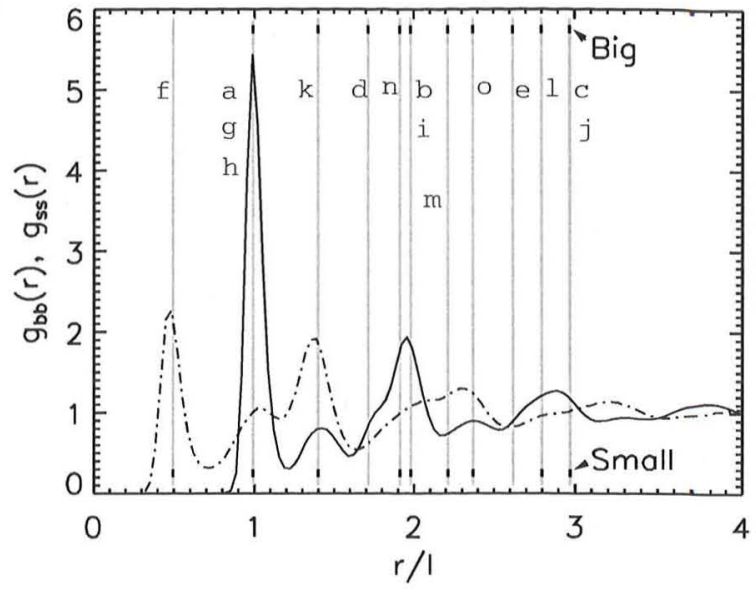

\begin{tabular}{|c|c|c|c|c|c|c|c|c|c|c|c|c|c|c|c|}
\hline Notation & a & b & c & d & e & f & g & h & i & j & k & l & m & n & o \\
\hline Length/a & 1 & 2 & 3 & $\sqrt{3}$ & $\sqrt{7}$ & $1 / 2$ & 1 & 1 & 2 & 3 & $\sqrt{2}$ & $2 \sqrt{2}$ & $\sqrt{5}$ & $\sqrt{2+\sqrt{3}}$ & $\sqrt{4+\sqrt{3}}$ \\
\hline
\end{tabular}

Fig. 5. Left: Basic crystal structures with highlighted unit cells: Big particles alone form hexagonal crystals, small particles alone arrange in chains. Mixtures of both species with a relative concentration of $\xi=1 / 2$ form SQ crystallites. The fourth structure is a combination of hexagonal and SQ with relative concentration of $\xi=1 / 3$ and has a rhombic unit cell. The shortest and therefore most significant lattice vectors and lengths are indicated as arrows. Lattice vectors that show up in several structures (e.g. vectors of SQ unit cell in rhombic structure) are not highlighted twice for reasons of clarity. Right: Pair correlation functions of big (solid) and small (dashed) particles for $\Gamma=674$ and relative concentration $\xi=42 \%$. Peaks are sharp and correlated between species. Grey bars correspond to the lengths of the shortest lattice vectors of the drawn crystal structures. The labels at the bar ends note which bar is affiliated to $g_{b b}(r)$ or $g_{s s}(r)$. All peaks and bars coincide one to one. Bottom: The table contains the calculated lengths of the indicated lattice vectors from ideal crystal structures and therefore the positions of the bars. The underlying lattice spacing a (hexagonal, SQ and rhombic) is taken from the position of the first maximum of $g_{b b}(r)$ at $a=0.99 \cdot l$ and for the chains the underlying spacing is taken as $a / 2$.

dynamically arrested state at $\Gamma=674$. The graph shows that all features in the pair correlation function are in excellent agreement with the simple crystal lattices drawn above: A hexagonal crystal of $100 \%$ big particles, chains of small particles and a SQ crystal of 50\% small and 50\% big particles. As mentioned above, small particles cannot form hexagonal $2 \mathrm{D}$ crystals, but only chains. This is confirmed by the missing peak in $g_{s s}(r)$ at $r / l=\sqrt{3} / 2$ (long diagonal distance of hexagonal rhomb) found in $g_{b b}(r)$ at $r / l=\sqrt{3}$. A fourth structure (Fig. 5, left) stems from combination of the hexagonal and SQ structure and can be described as rhombic with a threefold basis [16]. In this way the system may form periodic structures with a relative concentration of $\xi=1 / 3$. All shortest lattice vectors highlighted in the lattices are found in the pair correlation functions at the positions of the grey bars. The peaks in both correlation functions at $r / l=\sqrt{4+\sqrt{3}} \approx 2.39$ are statistical evidence for the rhombic structure. They cannot be explained by any of the other structures and as shown in Figure $4 \mathrm{E}$ they also occur in large grains.

\subsection{Dependence of local crystalline order on relative concentration}

In this section we address the question how the local crystal structure depends on the relative concentration $\xi$. In
Figure 6 we compare the pair correlation functions $g(r)$ of two samples with relative concentration $\xi=45 \%$ and $\xi=29 \%$ at strong supercooling $\Gamma=556$ and $\Gamma=529$, respectively. Due to a small shift of peak position in $r$ induced by the small particle fraction [15], the length scale $l=1 / \sqrt{\rho}_{\text {big }}$ of the sample with $\xi=29 \%$ was normalized by a factor of 1.04 to compare corresponding peaks.

We find that no additional peaks are showing up and no peak is vanishing. Only the relative contributions from the different underlying crystal structures are changed by the change in relative concentration. With decreasing $\xi$ the big particles show stronger order in the hexagonal phase compared to the SQ phase. This results in a relative decrease of the peaks in $g_{b b}(r)$ at $r / l=\sqrt{2}$ (SQ, peak $k$ in Fig. 5) and $r / l=\sqrt{4+\sqrt{3}} \approx 2.39$ (rhombic, peak $O$ in Fig. 5) and a strong relative increase of peaks at $r / l=\{1,2,3, \sqrt{3}, \sqrt{7}\}$ (hexagonal, peaks $a, b, c, d, e$ in Fig. 5).

The change in the configurations of the small particles as seen in $g_{s s}(r)$ indicates a relative increase of SQ order (peak $k$ at $r / l=\sqrt{2}$ in Fig. 5) compared to chain order (peak $f$ at $r / l=0.5$ ).

The comparison of both samples shows that the underlying crystal structures are not dependent on the relative concentration. Only the relative fraction of crystallites is 


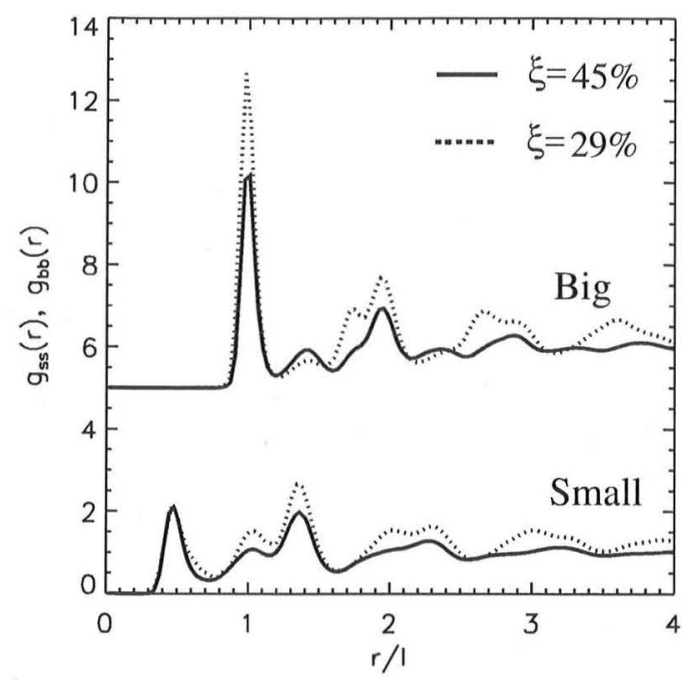

Fig. 6. Pair correlation functions $g(r)$ of only small (bottom) and only big (top) particles for two samples with different relative concentration (dashed: $\xi=29 \%$ at $\Gamma=529$, solid: $\xi=45 \%$ at $\Gamma=556$ ). For comparison of the peaks the $\xi=29 \%$ samples $\mathrm{r}$-axis was scaled with a factor of 1.04 . This is due to a peak shift induced by the higher fraction of small particles (partial clustering). The upper graph is shifted by 5 for reason of clarity. The peak heights change due to the relative fraction of different crystallite structures.

influenced when the amount of small particles is decreased: Hexagonal order of big particles is increased compared to SQ order, and SQ order of the small particles is increased compared to chain order.

\subsection{Dependence of orientational order on interaction parameter $\Gamma$}

The hexagonal crystal structure was already investigated in monodisperse systems [19]. The symmetry was investigated by sixfold bond order correlation function $G_{6}(r)$ which is sensitive to the different bond order decays and decay lengths in different phases [24]. It is remarkable that the hexagonal structure also appears in the binary mixture. The formation of tiny hexagonal crystallites is not suppressed completely by the surrounding heterogenous binary structure.

The square unit cell is a typical constituent occurring in the SQ structure as well as in the rhombic structure. Therefore we analyze the fourfold order for increasing interaction strength $\Gamma$ in the binary case using the fourfold bond order correlation function $G_{4}(r)$ defined as

$$
G_{4}(r)=\left\langle\left|\psi_{4}\left(\mathbf{r}_{i}\right) \psi_{4}^{*}\left(\mathbf{r}_{j}\right)\right|\right\rangle_{i j}
$$

with $r=\left|\mathbf{r}_{i}-\mathbf{r}_{j}\right|$ and $\psi_{4}\left(\mathbf{r}_{k}\right)=\frac{1}{N_{l}} \sum_{l} e^{i 4 \theta_{k l}}$, where the sum is carried out over all $l$ nearest neighbors and $\theta_{k l}$ is the angle between $\mathbf{r}_{k}-\mathbf{r}_{l}$ and a common reference axis. The result is shown in Figure 7. The bond order correlation function $G_{4}$ for big particles of a very fluid sample

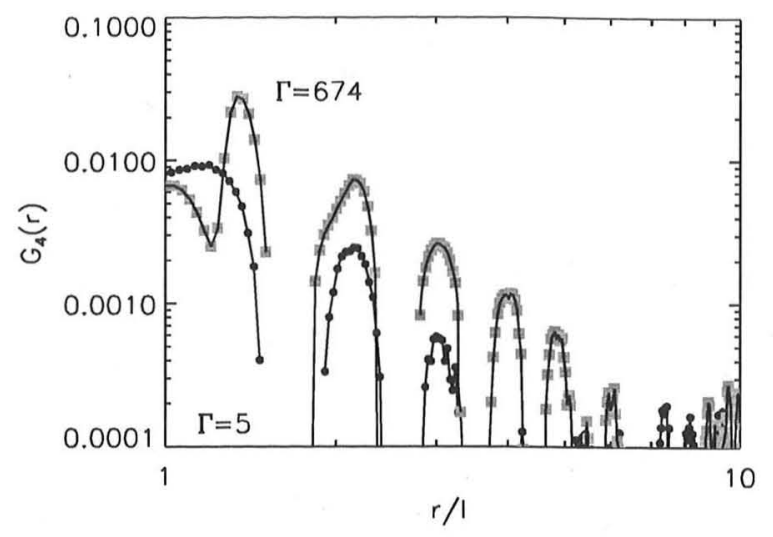

Fig. 7. Bond order correlation function $G_{4}$ for two indicated values of $\Gamma$ of just the big particles $(\xi=42 \%)$. Although the difference in supercooling is drastic, the decay length of fourfold orientation is not significantly increased. The system never exhibits any long-range order.

$(\Gamma=5)$ is compared with a strongly supercooled sample $(\Gamma=674) . G_{4}$ decays on the length scale of a few interparticle distances $l$ and shows no significant $\Gamma$-dependence. The oscillations of the curves result from the local order of the particles and reflect the pair correlation function $g_{b b}(r)$. Note the height ratio of the first two peaks in the curve at $\Gamma=674$. From the pair correlation function of the big particles in Figure 5 it is found that the distance $r / l=1$ is much more frequent than the distance $r / l=\sqrt{2}$. Here the corresponding peaks show the opposite behavior. This is understood by the degree of fourfold order connected with these distances. While $r / l=1$ corresponds to both hexagonal (sixfold) and SQ order (fourfold), the distance $r / l=\sqrt{2}$ is related exclusively to fourfold SQ order. This local ordering in hexagonal and SQ order significantly enhances the second peak in comparison to the first one.

As the system does not exhibit any long-range orientational order in the binary case we focus on local fourfold order. The explicit fraction of SQ unit cells found in the local order is discussed first. To compare this order for different parameters, three selection criteria for the occurrence of square unit cells are introduced:

1. Selection of configurations with 1 small particle surrounded by 4 big particles.

2. Fourfold local bond order parameter $\Psi$ of configurations selected in 1).

3. Bond length deviation parameter $b$ of configurations selected in 1).

As for the first criterion we check whether the four closest particles of a small particle are big ones. The fourfold bond order parameter of these configurations for the second criteria is defined as

$$
\Psi=\frac{1}{4} \sum_{N N}^{4} e^{i 4 \theta_{N N}},
$$

where $\theta_{N N}$ are the angles of the four bonds between the small bead in the center and the surrounding big particles 

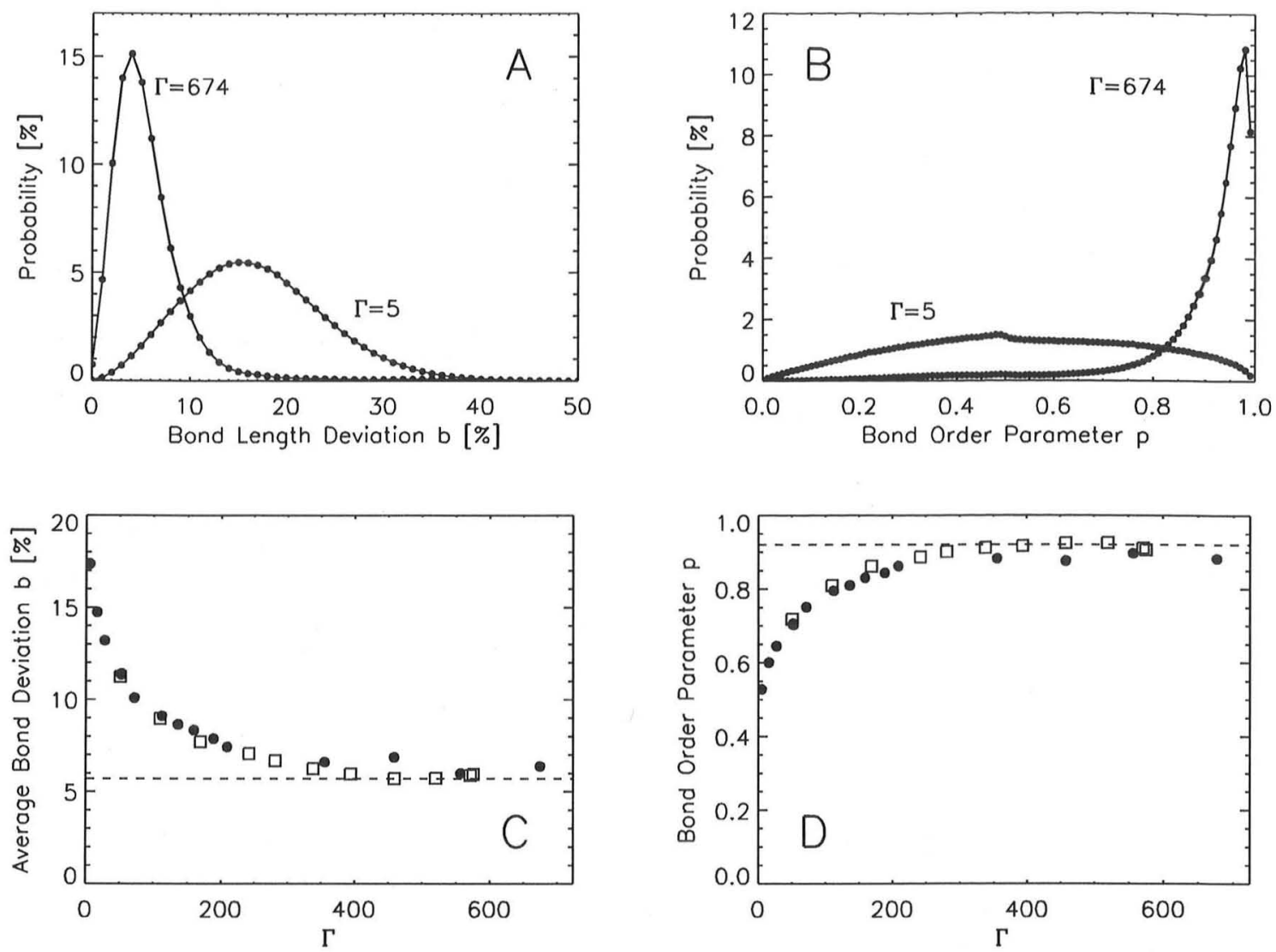

Fig. 8. Graph A: Histogram of bond length deviations averaged over the four bonds of quadrangular configurations (1 small bead in the center surrounded by 4 big beads) are shown for two different values of $\Gamma$. Graph B: Histogram of local bond order parameters for two values of $\Gamma$ calculated only for quadrangular configurations. Local fourfold order and average bond length are strongly enhanced with increasing $\Gamma$. Graph C: The average deviation of bond lengths in SQ configurations (4 big, $1 \mathrm{small})$ is shown versus $\Gamma$. Graph D: Bond order parameter $p=\sqrt{\Psi^{*} \Psi}$ of SQ configurations is plotted versus $\Gamma$. The dashed lines indicate the saturation values $b=5.7 \%$ and $p=0.92$. The symbols (open squares, filled circles) differ because two different samples were analyzed. Both samples have relative concentrations of $\xi \approx 45 \%$.

relative to a fixed reference. The bond order parameter $p=\sqrt{\Psi^{*} \Psi}$ is a measure for the local fourfold symmetry. For perfect right angles this parameter is $p=1$. All other configurations have lower values $p>0$.

To characterize a SQ square this criterion is not sufficient since also other configurations like a rhomb may have right angles. Therefore in the third criteria the bond length deviation parameter $b$ is introduced as

$$
b=\frac{1}{4} \sum_{N N}^{4} \frac{\left|l_{N N}-\bar{l}\right|}{\bar{l}}
$$

with the four bond lengths $l_{N N}$ and their average length $\bar{l}$. For a perfect rectangle (not only squares) this quantity becomes zero. All three criteria combined can be used as a local fourfold order parameter.

The graphs in Figure 8 show how sensitive these parameters vary when the interaction parameter $\Gamma$ is changed. A strong and continuous increase of order is found in both criteria. The upper graphs Figures $8 \mathrm{~A}$ and $\mathrm{B}$ show the distribution of both parameters $b$ and $p$ for two extreme values of $\Gamma$. For the fluid case $(\Gamma=5)$ the local order is very low as the distribution of $b$ is broad and centered around a value of about $15 \%$. At $\Gamma=674$ the distributions of $b$ and $p$ are strongly peaked which reflects the high local order. Average values of $b$ and $p$ saturate with increasing $\Gamma$ shown in Figures $8 \mathrm{C}$ and D. These saturation values are taken as selection limits to determine whether a quadrangular configuration is labelled as square unit cell. In Figure 9 these selected SQ configurations are highlighted in particular snapshots. Figure 10 shows the fraction of SQ unit cells normalized by the number of small particles (the maximum number of possible SQ configurations). A continuous increase in the fraction of SQ unit cells is observed indicating the tendency for crystallization. The measurements for two different samples agree well for $\Gamma<220$ but significantly deviate for larger values of $I$, although both samples had comparable relative concentrations.

Two reasons may explain this: i) The sample with less order for $\Gamma>220$ (filled circles) happened to have a slight total drift, while the other (open squares) was absolute 

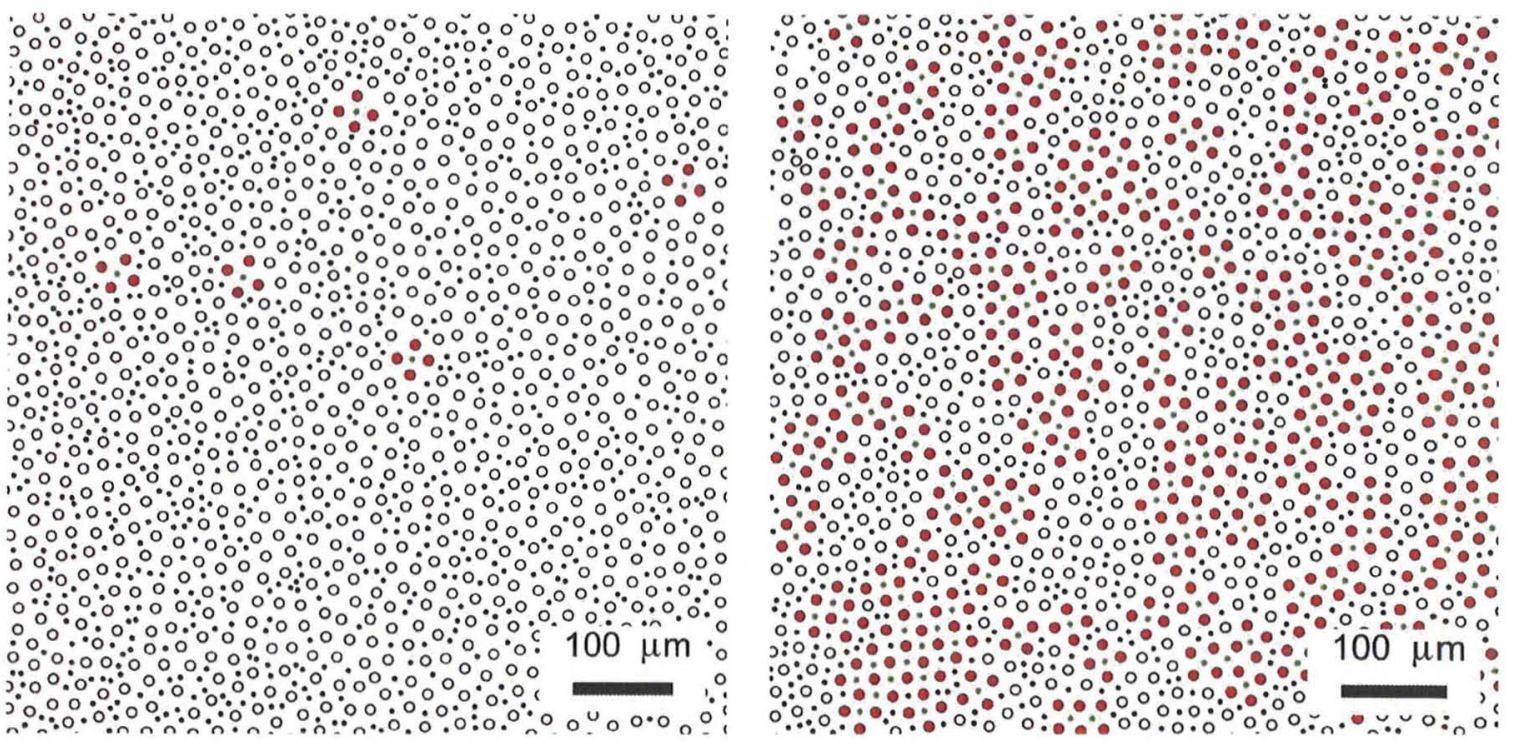

Fig. 9. Snapshots for $\Gamma=51$ (left) and $\Gamma=520$ (right). The highlighted particles have SQ unit cell configuration. The criteria of whether a quadrangular configuration is considered as a SQ unit cell is $b<5.7 \%$ and $p=\sqrt{\Psi^{*} \Psi}>0.92$, the saturation values taken from Figures $8 \mathrm{C}$ and D.

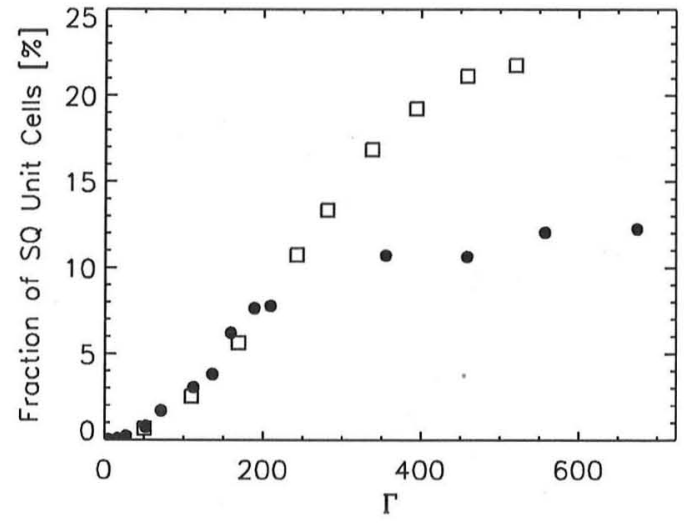

Fig. 10. Fraction of SQ unit cells versus $\Gamma$. The symbols (open squares, filled circles) differ because two different samples were analyzed (both $\xi \approx 45 \%$ ). For low $\Gamma$ both samples exhibit same amounts of SQ order. For high $\Gamma$, where the system is nonergodic, the individual local particle distribution and sample history plays a role. A continuous increase in local order is observed for decreasing system temperature.

quiescent. A small drift causes a non-negligible shear in the sample which may have lead to a reduction in local order. ii) The samples have different preparation histories, one is cooled down very slowly (open squares) whereas the other (filled circles) was cooled down rapidly and the field of view was varied. Therefore the coincidence at low $\Gamma$ and the deviation at high $\Gamma$ can be explained by the nonergodicity of the system at these strongly supercooled states on the timescale of measurement. Whereas for lower $\Gamma$ the fraction of unit cells seems to be an equilibrium quantity, for higher $I$ it depends strongly on the history and individual composition of the sample.

\section{Conclusion}

The structure of our model 2D binary glass former shows no long-range order but has a clear underlying substructure, which originates from the system's tendency to crystallize into a small set of crystal structures: Hexagonal order of big particles, chains of small particles, simple quadratic (SQ) with a local relative concentration of $\xi=1 / 2$ and rhombic with a local relative concentration of $\xi=1 / 3$. This was shown on the one hand by the appearance of extended stable crystal grains and, on the other hand, by the peaks of the pair correlation functions which are in agreement with these lattice structures. Changing the relative concentration of the mixture does not affect the peak structure in the pair correlation functions, but modifies the relative peak heights. This demonstrates, that the crystal structures do not change by themselves, but their relative contribution to the globally disordered state. The rapid decay of the fourfold bond order correlation function reveals that increasing the interaction parameter $\Gamma$ does not significantly enhance long-range order but strongly enhances local fourfold order, as found in the local bond order statisties. Different selection criteria were introduced to probe the amount of square unit cells for a given interaction $\Gamma$. A continuous increase with some indication of a change in slope near $\Gamma \approx 50$ is found in Figure 10 which might indicate an underlying phase transition. The saturation at high $\Gamma$ values depends on the individual history and local composition of the sample.

Beside the investigated structures there is a whole bunch of other even more exotic crystal lattices that are predicted to be stable [16] especially for higher relative concentrations of small particles. In particular the structures collectively described as "chains" in this work are expected to be identified as pieces of crystalline order with high relative concentration $\xi$. 
Of special interest is the connection of the found structures to local dynamics and how local crystallinity is related to dynamic heterogeneity [11]. From the stability of larger crystal grains it is expected that regions with high crystallinity strongly contribute to slow down the systems dynamics.

We thank P. Dillmann and U. Gasser for ideas and fruitful discussion. This work was supported by the DFG (Deutsche Forschungsgemeinschaft) in the frame of Sonderforschungsbereich 513 project B6 and the Sonderforschungsbereich Transregio 6 project $\mathrm{C} 2$.

\section{References}

1. P. Pusey, W. van Megen, Nature 320, 340 (1986).

2. F. Ebert, T. Thurn-Albrecht, Macromolecules 36, 8685 (2003).

3. T. Scopigno, J.-B. Suck, R. Angelini, F. Albergamo, G. Ruocco, Phys. Rev. Lett. 96, 135501 (2006).

4. U. Bengtzelius, G. Götze, A. Sjölander, J. Phys. C 17, 5915 (1984).

5. D. Reichman, P. Charbonneau, Cond.Mat. arXiv: 0511407v1 (2005).

6. E. Weeks, J.C. Crocker, A. Levitt, A. Schofield, D. Weitz, Science 287, 627 (2000).

7. A. Widmer-Cooper, P. Harrowell, J. Phys.: Condens. Matter 17, 4025 (2005).

8. A. Widmer-Cooper, P. Harrowell, H. Fynewever, Phys. Rev. Lett. 93, 135701 (2004).

9. P. Harrowell, Nature Phys. 2, 157 (2006)

10. H. Shintani, H. Tanaka, Nature Phys. 2, 200 (2006).
11. H. König, R. Hund, K. Zahn, G. Maret, Eur. Phys. J. E 18, 287 (2005).

12. D. Perera, P. Harrowell, Phys. Rev. E 59, 5721 (1999).

13. M. Bayer, J. Brader, F. Ebert, M. Fuchs, E. Lange, G. Maret, R. Schilling, M. Sperl, J. Wittmer, Phys. Rev. E 76, 011508 (2007).

14. N. Hoffmann, F. Ebert, C. Likos, H. Loewen, G. Maret, Phys. Rev. Lett. 97, 078301 (2006).

15. N. Hoffmann, C. Likos, H. Löwen, J. Phys.: Condens. Matter 18, 10193 (2006)

16. L. Assoud, R. Messina, H. Löwen, Cond.Mat. arXiv: 0706.2311v2 (2007).

17. F. Scheffler, P. Maass, J. Roth, H. Stark, Eur. Phys. J. B 42, 85 (2004).

18. H. König, Europhys. Lett. 71, 838 (2005).

19. K. Zahn, R. Lenke, G. Maret, Phys. Rev. Lett. 82, 2721 (1999).

20. H.H.v. Grünberg, P. Keim, K. Zahn, G. Maret, Phys. Rev. Lett. 93, 255703 (2004)

21 . To reach the same value $\Gamma_{s}$ in a monodisperse sample with $\chi_{s}$ and $l_{s}$ compared to a monodisperse case with $\chi_{b}$ and $l$ a magnetic field $H_{s}=H_{b} \cdot\left(l_{s} / l\right)^{3 / 2} \cdot \chi_{b} / \chi_{s}$ has to be applied. The interparticle distance of small particles $l_{s}=0.5 \cdot l$ (first peak pair correlation function $g_{s s}(r)$ ) and $\chi_{b} / \chi_{s} \approx 10$ lead to a magnetic field $H_{s}$ stronger by a factor 3.54. In our binary case this would approximately correspond to $\Gamma=3.54^{2} \cdot 60 \approx 750$ which is above the used experimental values.

22. In "Classical Nucleation Theory" the formation of a critical nucleus is assumed to happen spontaneously from the liquid phase directly into a grain of the solid phase overcoming a potential barrier consisting of surface minus bulk energy.

23. S. Auer, D. Frenkel, Nature 409, 1020 (2001).

24. P. Keim, H.H.v. Grünberg, G. Maret, Phys. Rev. E 75, 031402 (2003). 